\title{
On the Transverse-Momentum Distribution of Secondary Particles from High-Energy Collisions.
}

\author{
F. C. JONES \\ Laboratory for Theoretical Studies, Goddard Space-Flight Center \\ National Aeronautics and Space Administration - Greenbelt, Md.
}

(ricevuto il 17 Giugno 1966)

In a recent paper FRIEDLÄNDER (1) states that only a Boltzmann-type distribution in transverse momentum of particles emitted in a high-energy collision is compatible with axial symmetry about the collision axis. He refers to a paper by ALy, KAPLON and Shen $\left({ }^{2}\right)$ for the proof of this statement. It is the purpose of this note to point out that the "proof" of Aly, Kaplon and Shen is in error and that in fact the Boltzmanntype distribution does not follow from axial symmetry alone.

What this argument essentially says is that a function of the type

$$
\text { (1) } N\left(p_{t}\right) \mathrm{d} p_{t}=2 \alpha p_{t} \exp \left[-\alpha p_{t}^{2}\right] \mathrm{d} p_{t} \text {, }
$$

is the only function that has axial symmetry. This is, on the face of it, untrue because any distribution that is a function of $p_{t}$ alone and not a function of the azimuth angle $\varphi$ has axial symmetry. The fault lies in eq. (1) of Aly, Kaplon and Shen which the authors state to be

(1) E. M. FRIEDL ̈NDER: Nuovo Cimente, 41, $417(1966)$.

(2) H. H. ALY, M. F. KAPLON and M. L. Sher: Nuovo Cimento, 81, 905 (1964). an expression of axial symmetry. This equation,

$$
N\left(p_{t}\right)=f\left(p_{x}\right) \cdot f\left(p_{y}\right)=F\left(p_{t}^{2}\right),
$$

where

$$
p_{t}^{2}=p_{x}^{2}+p_{y}^{2},
$$

is, in fact, not just a statement of axial symmetry (this is contained in the state. ment that it is a function of $p_{t}$ alone) but is in addition a statement of sta. tistical independence of the distribution in $p_{x}$ and $p_{y}$. This is quite a bit more than simple symmetry. It is well known that when one demands symmetry and independent distribution in the rectangular co-ordinates one is led automatically to a Boltzmann-type distribution. In fact it is just this combination of assumptions that led Maxwell to his famous velocity distribution law, and it is the assumption of independence that is considered to be the weakest part of his derivation $\left(^{3}\right)$.

$\left({ }^{3}\right)$ J. Jgans: An Introduction to the Finetic Teorhy of Gases, Cambridge University Press, Cambridge (1948), Appendix I, p. 296. 